\title{
Trasplante de materia fecal en pacientes octogenarios con infección recurrente por Clostridioides difficile
}

\author{
Rodrigo Quera', Rocío Sedano², Ricardo Espinoza' , y Daniela Rivera ${ }^{1}$
}

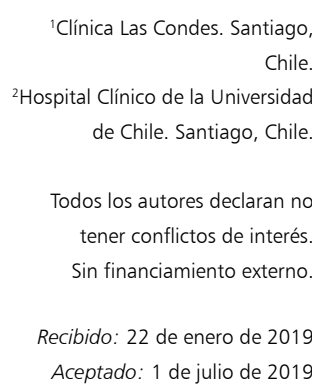

Correspondencia a: Rodrigo Quera rquera@clc.cl

\section{Transplantation of fecal matter in octogenarian patients with recurrent Clostridioides difficile infection}

Clostridioides (formerly Clostridium) difficile infection has become a major health problem due to the increase in its incidence, severity, and recurrence. In this last scenario, age over 65 has been associated with a more unfavorable evolution. Risk factors such as the presence of altered immunity, comorbidities, malnutrition, polypharmacy, and changes in the intestinal microbiota would explain this higher risk in this group of patients. On the other hand, fecal microbiota transplantation (FMT) is an effective strategy in the treatment of recurrent Clostridioides difficile infection when standard therapy fails. Recently published guidelines suggest that this strategy can be used from the second recurrence. However, few studies have evaluated the results of the FMT in patients over 65 years old, and for our knowledge, there is limited national experience in this group of patients. We present two cases of TMF in octogenarian patients with a recurrent infection due to Clostridioides difficile, with satisfactory recovery at the long term.

Keywords: Clostridium difficile; Clostridioides difficile; fecal microbiota transplantation; elderly; comorbidity

Palabras claves: Clostridium difficile; Clostridioides difficile; trasplante de microbiota fecal; adulto mayor; comorbilidad.

\section{Introducción}

$\longrightarrow$ lostridioides (previamente Clostridium) difficile (C. difficile) es una bacteria anaerobia, gram positiva, productora de esporas, que puede encontrarse como parte de la microbiota colónica habitual. Ante determinadas circunstancias, como el uso de antimicrobianos que alteran el microambiente intestinal, ciertos factores de virulencia de $C$. difficile, como la toxina A glucosiltransferasa y toxina $\mathrm{B}$, pueden producir injuria en el epitelio intestinal. Este daño desencadena una respuesta inflamatoria aguda secundaria en la mucosa colónica, con un infiltrado celular de predominio polimorfonuclear neutrófilo, y formación de pseudomembranas. La traducción clínica del cuadro descrito es variable, desde una diarrea leve, hasta casos graves de riesgo vital, tales como la colitis pseudomembranosa y el megacolon tóxico ${ }^{1-4}$.

Existen múltiples factores de riesgo asociados a infección por $C$. difficile (ICD), siendo uno de ellos, la edad mayor de 65 años (Tabla 1$)^{5-9}$. Lessa y cols. reportan una incidencia de ICD ocho veces mayor y una tasa de recurrencia de 3,5 a 10 veces mayor en mayores de 65 años, comparado con una población más joven ${ }^{10}$. Otros estudios también han confirmado como factor de riesgo de recurrencia la edad, el antecedente de ICD el año previo, entre otros ${ }^{11}$. El reporte nacional de estadísticas vitales de los CDC señala que más de $90 \%$ de la mortalidad relacionada a ICD ocurre en mayores de 65 años ${ }^{12}$. Un estudio reciente confirma que la edad mayor de 65 años así como también, la presencia de co-morbilidades serían factores de riesgo de mortalidad asociada a la $\mathrm{ICD}^{13}$.

En contexto de la disminución de la respuesta a la terapia antimicrobiana en ICD recurrente, y a la marcada alteración de la microbiota colónica posterior al uso de antibacterianos, es que se plantea el uso del trasplante de microbiota fecal (TMF) desde un donante sano, como alternativa terapéutica, en pacientes que presentan un segundo episodio de recurrencia de $\mathrm{ICD}^{14}$.

El primer TMF fue realizado el año 1958 por Eiseman y cols., mediante una infusión de materia fecal por enema en cuatro pacientes que presentaron un cuadro de diarrea fulminante asociada a antibacterianos ${ }^{15}$. Posteriormente diferentes estudios han confirmado su efectividad con tasas de respuesta entre $81-100 \%{ }^{16-21}$. Pese a ello, solo algunos estudios han confirmado que esta estrategia terapéutica puede ser utilizada en pacientes mayores de 65 años manteniendo tasas de respuesta adecuadas ${ }^{22,23}$. Recientemente, dos series nacionales han demostrado la efectividad del TMF; sin embargo, en ambos estudios solo se incluyó un paciente mayor de 65 años, con un seguimiento máximo de siete meses ${ }^{24,25}$.

En contexto del aumento de la población adulta mayor y de la mayor frecuencia de ICD en pacientes sobre 65 años, se presentan a continuación dos casos 
clínicos en pacientes octogenarios que en contexto de una ICD recurrente, fueron manejados de manera exitosa con el TMF.

\section{Casos clínicos}

\section{Caso 1}

Mujer de 82 años, con antecedentes de un carcinoma basocelular y una sinusitis aguda tratada con moxifloxacina por siete días. Quince días post-tratamiento antimicrobiano inició un cuadro de diarrea con reacción en cadena de la polimerasa (RPC), Genexpert ${ }^{\circledR}$, que detectó C. difficile, siendo tratada con metronidazol $500 \mathrm{mg}$ cada $8 \mathrm{~h}$ por 14 días. A los siete días de haber completado el tratamiento, la paciente presentó una recaída clínica con detección de CD por RPC y toxina, iniciándose terapia con vancomicina vía oral $125 \mathrm{mg}$ cada $6 \mathrm{~h}$ por 14 días, con posterior retiro progresivo escalonado del antibacteriano. A los cinco días de su término presentó un nuevo cuadro de deposiciones líquidas, fiebre y distensión abdominal con un estudio de toxina y RPC positivo a $C$. difficile. En el contexto de una segunda recurrencia, se decidió realizar un TMF cuyo donante fue la hija de la paciente. El procedimiento se realizó vía colonoscópica con $150 \mathrm{~g}$ de heces $/ 500$ cc solución fisiológica al 0,9\%. La solución se instiló desde el íleon terminal hasta el colon descendente proximal. La evolución posterior al TMF fue favorable, manteniéndose asintomática después de 24 meses del procedimiento.

\section{Caso 2}

Mujer de 81 años, con antecedente de episodios de diverticulitis recurrente. Inició un cuadro de dolor abdominal en la fosa ilíaca izquierda, diagnosticándose una diverticulitis aguda no complicada del sigmoides, por lo que recibió terapia con ciprofloxacina por ocho días sin respuesta, y luego ceftriaxona y metronidazol intravenoso por 14 días. En contexto de la persistencia de dolor en fosa ilíaca izquierda, y presencia de deformación a nivel del sigmoides secundaria a múltiples recurrencias de diverticulitis, se decidió realizar una sigmoidectomía. A las 48 h de la cirugía, la paciente inició un cuadro de deposiciones líquidas abundantes. El estudio detectó $C$. difficile por técnica de RPC, por lo que inició terapia con metronidazol $500 \mathrm{mg}$ tres veces al día por 10 días con una respuesta parcial, disminuyendo la frecuencia defecatoria de 20 a seis episodios diarios manteniendo el compromiso del estado general, por lo que se decidió realizar un cambio de estrategia terapéutica a vancomicina $125 \mathrm{mg}$ cuatro veces al día vía oral, con retiro progresivo (seis semanas). Tras cinco días de concluida la terapia, la paciente inició nuevamente deposiciones líquidas con frecuencia de hasta 25 episodios al día, con detección de
Tabla 1. Factores de riesgo asociados a infección por Clostridioides difficile (ICD) ${ }^{5-9}$

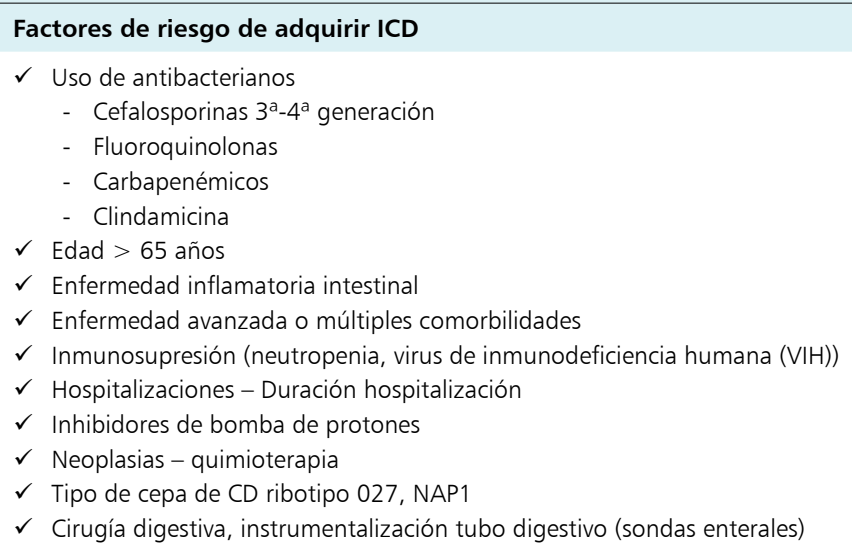

\section{Factores de riesgo de mortalidad asociada a ICD}

$\checkmark$ Edad $>65$ años

$\checkmark$ Leucocitosis

$\checkmark$ Falla renal aguda

$\checkmark$ Co-morbilidades

$\checkmark$ Hipoalbuminemia

$\checkmark$ Cepa CD ribotipo 027

C. difficile por estudio de toxina y RPC, iniciándose nuevamente vancomicina en un esquema de retiro progresivo, asociado a rifaximina por 20 días, con respuesta favorable. Sin embargo, nuevamente a los 10 días de completado el tratamiento aumentó la frecuencia defecatoria, nuevamente con una RPC positiva para C. difficile. En el contexto de una segunda recurrencia se realizó un TMF, cuyo donante fue su hijo. El procedimiento se realizó vía colonoscópica con $73 \mathrm{~g}$ de heces/350 cc solución fisiológica al 0,9\%. La solución se instiló desde el íleon terminal hasta el colon descendente proximal. La evolución posterior al TMF ha sido favorable, manteniéndose asintomática después de 10 meses del procedimiento.

\section{Discusión}

Si bien, cada vez existen más publicaciones respecto de la efectividad del TMF en el tratamiento de la recurrencia por $C$. difficile, aun existen pocos reportes de su uso en pacientes adultos mayores. Uno de los estudios iniciales que comparó la administración de materia fecal vía nasoenteral vs. 14 días de vancomicina, demostró una mejoría en $94 \%$ vs. $31 \%$ de los pacientes, respectivamente ${ }^{16}$. Otro estudio aleatorizó a 39 pacientes a TMF $(n=20)$ o vancomicina $(\mathrm{n}=19)$, realizando la administración vía colonoscópica, obteniendo una resolución sintomática en $90 \%$ de los pacientes con TMF, vs. $26 \%$ en el grupo que 
recibió vancomicina ${ }^{17}$. Ambos estudios fueron realizados en pacientes mayores de 70 años.

Recientemente, un estudio prospectivo de cohorte observacional siguió a 34 pacientes mayores de 60 años con ICD recurrente o con falta de respuesta a la terapia antimicrobiana. De los pacientes incluidos, 23 (68\%) fueron tratados con vancomicina y $11(32 \%)$ recibieron TMF. La edad promedio fue de 82 años ( $\mathrm{R}=60-94$ años), con 28 pacientes ( $82 \%$ ) mayores de 75 años. Al tercer día de tratamiento, se evidenció una mejor respuesta clínica en el grupo TMF vs control (90 vs 39\%; $p=0,006$ ), aunque sin diferencias en la estadía hospitalaria. En el sub-análisis del grupo de pacientes mayores de 75 años (ocho en el grupo TMF y 19 en el grupo control), se observó que los del grupo TMF tuvieron un mejor resultado clínico al tercer día de tratamiento $(86 \%$ vs $42 \% ; p=0,048)$ y mayor sobrevida a mediano plazo (12 vs tres meses; $\mathrm{p}=0,031)^{22}$.

Otro estudio prospectivo observacional en 29 pacientes, 23 mujeres y 6 varones, con edad promedio de 80 años $(\mathrm{R}=70-91$ años $)$, todos asociados a uso previo de antibacterianos, fueron sometidos a TMF vía yeyunal y colónica combinadas, tras falla a tres o más esquemas antimicrobianos. El tiempo medio transcurrido para la resolución de los síntomas digestivos (dolor abdominal, distensión y diarrea) fue de cuatro días ( $\mathrm{R}=1-10$ días $)$, todos los pacientes tuvieron una regresión parcial de los síntomas. Al momento de completar el período de seguimiento (25,4 $\pm 12,8$ meses), ningún paciente presentó recurrencia de la ICD. Otro hallazgo interesante, fue el análisis genómico que sugirió que la diversidad global de la microbiota fecal aumentó considerablemente posterior al TMF, con una disminución del filum Proteobacteria ${ }^{23}$.

Estudios realizados por Youngster y cols. utilizando el TMF por medio de cápsulas congeladas en pacientes con cuadros recurrentes o refractarios de ICD, han obtenido buenos resultados, alcanzando tasas de respuesta sobre $80 \%$ tras un primer tratamiento con TMF, y hasta $90 \%$ tras un segundo curso terapéutico ${ }^{19,26,27}$.

La población adulta mayor es de especial interés, dado que se asocia a una mayor susceptibilidad de presentar una ICD y recurrencias. Factores como la presencia de una inmunidad alterada (disminución del número y función de los linfocitos T y B), co-morbilidad, malnutrición, y polifarmacia, con el uso a veces excesivo de antibacterianos e inhibidores de la bomba de protones, han sido asociado a este mayor riesgo. ${ }^{28-32}$. Otro factor, son los cambios en la microbiota fecal asociados a la edad, lo cual podría predisponer a este grupo a un mayor riesgo de ICD y una evolución más desfavorable ${ }^{33}$.

Algunos estudios han señalado que pacientes adultos mayores institucionalizados o con antecedentes de ICD, presentan una disminución de bifidobacterias en su microbiota fecal, a diferencia de aquellos adultos mayores sanos o población menor de 65 años ${ }^{34-36}$. Así mismo, los pacientes mayores de 60 años presentarían una disminución de la abundancia relativa del filum Actinobacteria, y de la diversidad de la flora comensal anaerobia como Bacteroides y bifidobacterias, y cambio de la microbiota dominante ( $>10^{9}$ unidades formadoras de colonias (UFC)/g) por Firmicutes y Bacteroidetes ${ }^{37-40}$.

Dada la importancia del tipo de microbiota, la identificación de un donante fecal sano es el paso inicial esencial para obtener un TMF exitoso. Dentro de las características que se requieren cumplir para calificar como donante sano, están el tener $\geq 18 \mathrm{y} \leq 60$ años, con un índice de masa corporal entre $\geq 18 \mathrm{y} \leq 30 \mathrm{~kg} / \mathrm{m}^{2}$. ${ }^{41,42}$. Además, el donante debe ser estudiado ampliamente, descartando factores de riesgo y enfermedades infecciosas. En nuestros dos pacientes, se cumplieron las recomendaciones mencionadas en diferentes publicaciones ${ }^{15,24,42}$.

En los casos clínicos expuestos, ambas pacientes fueron octogenarias con cuadros recurrentes de ICD, uno moderado y el otro grave, que lograron un tratamiento efectivo con TMF, sin nuevos episodios de recurrencia a la fecha. En ambas pacientes el donante fue un familiar directo (hijo), y el método utilizado fue la colonoscopía, sin presentar eventos adversos asociados al procedimiento. Al igual que otras series nacionales en TMF, la evolución post-TMF en pacientes mayores de 75 años fue favorable, manteniéndose libre de recurrencias durante un mayor período de seguimiento (10 y 24 meses; respectivamente $)^{24,25}$.

\section{Resumen}

La infección por Clostridioides (previamente Clostridium) difficile se ha convertido en un problema de salud importante debido al aumento en su incidencia, gravedad y recurrencia. En este último escenario, una edad mayor de 65 años ha sido asociada a una evolución más desfavorable. Factores de riesgo como la presencia de una inmunidad alterada, co-morbilidades, malnutrición, polifarmacia y cambios en la microbiota intestinal explicarían este mayor riesgo a mayor edad. El trasplante de microbiota fecal (TMF) es una estrategia efectiva en el tratamiento de la infección recurrente por Clostridioides difficile cuando la terapia estándar fracasa. Guías publicadas recientemente sugieren que esta estrategia puede ser utilizada a partir de la segunda recurrencia. Sin embargo, escasos estudios han evaluado los resultados del TMF en pacientes mayores de 65 años y para nuestro conocimiento existe una escasa experiencia nacional en este grupo de pacientes. Presentamos dos casos de TMF en pacientes octogenarios con una infección recurrente por Clostridioides difficile, con una evolución satisfactoria a largo plazo. 


\section{Referencias bibliográficas}

1.- Tauxe W M, Haydek J P, Rebolledo P A, Neish E, Newman K L, Ward A, et al. Fecal microbiota transplant for Clostridium difficile infection in older adults. Therap Adv Gastroenterol 2016; 9: 273-81. doi: $10.1177 / 1756283 X 15622600$.

2.- Bartlett J G, Chang T W, Gurwith M, Gorbach S L, Onderdonk A B. Antibiotic-associated pseudomembranous colitis due to toxinproducing clostridia. N Engl J Med 1978; 298: 531-4. doi: 10.1056/NEJM197803092981003.

3.- Pruitt R N, Lacy D B. Toward a structural understanding of Clostridium difficile toxins A and B. Front Cell Infect Microbiol 2012; 2: 28. doi: 10.3389/fcimb.2012.00028.

4.- McDonald L C, Coignard B, Dubberke E, Song X, Horan T, Kutty P K. Recommendations for surveillance of Clostridium difficile-associated disease. Infect Control Hosp Epidemiol 2007; 28:140-5. doi: 10.1086/511798.

5.- McDonald L C, Gerding D N, Johnson S, Bakken J S, Carroll K C, Coffin S E, et al. Clinical Practice Guidelines for Clostridium difficile Infection in Adults and Children: 2017 Update by the Infectious Diseases Society of America (IDSA) and Society for Healthcare Epidemiology of America (SHEA). Clin Infect Dis 2018; 66: e1-e48. doi: 10.1093/cid/cix1085.

6.- Pépin J, Saheb N, Coulombe M A, Alary M E, Corriveau M P, Authier S, et al. Emergence of fluoroquinolones as the predominant risk factor for Clostridium difficile-associated diarrhea: a cohort study during an epidemic in Quebec. Clin Infect Dis 2005; 41:1254-60. doi: $10.1086 / 496986$

7.- Johnson S, Samore M H, Farrow K A, Killgore G E, Tenover F C, Lyras D, et al. Epidemics of diarrhea caused by a clindamycin-resistant strain of Clostridium difficile in four hospitals. N Engl J Med 1999; 341:1645-51. doi:10.1056/ NEJM199911253412203.

8.- Dethlefsen L, Huse S, Sogin M L, Relman D A. The pervasive effects of an antibiotic on the human gut microbiota, as revealed by deep $16 \mathrm{~S}$ rRNA sequencing. PLoS Biol 2008; 6:e280. doi: 10.1371/journal.pbio.0060280.

9.- Kelly CP, LaMont JT. Clostridium difficilemore difficult than ever. N Engl J Med 2008; 359: 1932-40. doi: 10.1056/NEJMra0707500

10.- Lessa FC, Mu Y, Bamberg WM, Beldavs ZG, Dumyati GK, Dunn JR, et al. Burden of Clostridium difficile infection in the United States. N Engl J Med 2015; 372: 825-34. doi: 10.1056/NEJMoa1408913.

11.- Cobo J, Merino E, Martínez C, Cózar-Llistó A, Shaw E, Marrodán T, et al. Prediction of recurrent Clostridium difficile infection at the bedside: the GEIH-CDI score. Int J Antimicrob Agents 2018; 51: 393-8. doi: 10.1016/j. ijantimicag.2017.09.010.
12.- Minino A M, Xu J, Kochanek K D. National Vital Statistics Reports. Vol 59, No 2. Centers for Disease Control and Prevention (CDC). http://www.cdc.gov/nchs/data/nvsr/nvsr59/ nvsr59_02.pdf.

13.- Cózar A, Ramos-Martínez A, Merino E, Martínez-García C, Shaw E, Marrodán T, et al. High delayed mortality after the first episode of Clostridium difficile infection. Anaerobe 2019; 57: 93-8. doi: 10.1016/j.anaerobe.2019.04.004.

14.- Cammarota G, Ianiro G, Tilg H, RajilićStojanović M, Kump P, Satokari R, et al. European consensus conference on faecal microbiota transplantation in clinical practice. Gut 2017; 66: 569-80. doi: 10.1136/ gutjnl-2016-313017.

15.- Eiseman B, Silen W, Bascom G S, Kauvar A J. Fecal enema as an adjunct in the treatment of pseudomembranous enterocolitis. Surgery 1958; 44: 854-9.

16.- van Nood E, Vrieze A, Nieuwdorp M, Fuentes S, Zoetendal E G, de Vos W M, et al. Duodenal infusion of donor feces for recurrent Clostridium difficile. N Engl J Med 2013; 368: 407-15. doi: 10.1056/NEJMoa1205037.

17.- Cammarota G, Masucci L, Ianiro G, Bibbò $S$, Dinoi G, Costamagna G, et al. Randomised clinical trial: faecal microbiota transplantation by colonoscopy versus vancomycin for the treatment of recurrent Clostridium difficile infection. Aliment Pharmacol Ther 2015; 41: 835-43. doi: 10.1111/apt.13144.

18.- Lee C H, Steiner T, Petrof E O, Smieja M, Roscoe D, Nematallah A, et al. Frozen vs fresh fecal microbiota transplantation and clinical resolution of diarrhea in patients with recurrent Clostridium difficile infection: a randomized clinical trial. JAMA 2016; 315: 142-9. doi: 10.1001/jama.2015.18098.

19.- Youngster I, Sauk J, Pindar C, Wilson R G, Kaplan J L, Smith M B, et al. Fecal microbiota transplant for relapsing Clostridium difficile infection using a frozen inoculum from unrelated donors: A randomized, open-label, controlled pilot study. Clin Infect Dis 2014; 58: 1515-22. doi: 10.1093/cid/ciu135.

20.- Kelly C R, Khoruts A, Staley C, Sadowsky M J, Abd M, Alani M, et al. Effect of fecal microbiota transplantation on recurrence in multiply recurrent Clostridium difficile infection: a randomized trial. Ann Intern Med 2016; 165: 609-16. doi: 10.7326/M16-0271.

21.- Quraishi M N, Widlak M, Bhala N, Moore D, Price M, Sharma N, et al. Systematic review with meta-analysis: the efficacy of faecal microbiota transplantation for the treatment of recurrent and refractory Clostridium difficile infection. Aliment Pharmacol Ther 2017; 46: 479-93. doi: 10.1111/apt.14201.

22.- Friedman-Korn T, Livovsky D M, Maharshak N, Aviv Cohen N, Paz K, Bar-Gil Shitrit A, et al. Fecal transplantation for treatment of
Clostridium difficile infection in elderly and debilitated patients. Dig Dis Sci. 2018; 63: 198 203. doi: 10.1007/s10620-017-4833-2.

23.- Girotra M, Garg S, Anand R, Song Y, Dutta $\mathrm{S} \mathrm{K}$. Fecal microbiota transplantation for recurrent Clostridium difficile infection in the elderly: long-term outcomes and microbiota changes. Dig Dis Sci. 2016; 61: 3007-15. doi: 10.1007/s10620-016-4229-8.

24.- Quera R, Ibáñez P, Simian D, Rivera D, Acuña G, Espinoza R. Fecal microbiota transplantation through colonoscopy for Clostridium difficile recurrent infection. Report of eight cases. Rev Med Chil 2018; 146: 859-66. doi: 10.4067/ s0034-98872018000800823.

25.- Cruz R, Monroy H, Flandez J, Pérez C, Álvarez-Lobos M, Hernandez-Rocha C. Practical clues for a fecal microbiota transplantation by colonoscopy for recurrent Clostridium difficile infection. Experience in a University center. Rev Chilena Infectol 2018; 35: 566-73. doi: 10.4067/s071610182018000500566

26.- Youngster I, Mahabamunuge J, Systrom H, Sauk J, Khalili H, Levin J, et al. Oral, frozen fecal microbiota transplant (FMT) capsules for recurrent Clostridium difficile infection. BMC Med 2016; 14: 134. doi 10.1186/s12916-0160680-9.

27.- Youngster I, Russell G, Pindar C, Ziv-Baran T, Sauk J, Hohmann EL. Oral, capsulized, frozen fecal microbiota transplantation for relapsing Clostridium difficile infection. JAMA 2014; 312: 1772-8. doi:10.1001/jama.2014.13875.

28.- Yoshikawa T T, Norman D C. Geriatric infectious diseases: current concepts on diagnosis and management. J Am Geriatr Soc 2017; 65: 631-41. doi: 10.1111/jgs.14731.

29.- Zheng B, Han S, Takahashi Y, Kelsoe G. Immunosenescence and germinal center reaction. Immunol Rev 1997; 160: 63-77.

30.- Romeo J, Wärnberg J, Pozo T, Marcos A. Physical activity, immunity and infection. Proc Nutr Soc 2010; 69: 390-9. doi: 10.1017/ S0029665110001795.

31.- Naylor K, Li G, Vallejo A N, Lee W W, Koetz K, Bryl E, et al. The influence of age on $\mathrm{T}$ cell generation and TCR diversity. J Immunol 2005; 174: 7446-52.

32.- Rodríguez C, Korsak N, Taminiau B, Avesani V, Van Broeck J, Delmée M, et al. Clostridium difficile infection in elderly nursing home residents. Anaerobe 2014; 30:184-7. doi: 10.1016/j.anaerobe.2014.08.007.

33.- Zapata H J, Quagliarello V J. The microbiota and microbiome in aging: potential implications in health and age-related diseases. J Am Geriatr Soc 2015; 63: 776-81. doi: 10.1111/jgs. 13310 .

34.- Hopkins M J, Macfarlane G T. Changes in predominant bacterial populations in human faeces with age and with Clostridium difficile 
infection. J Med Microbiol 2002; 51: 448-54. doi:10.1099/0022-1317-51-5-448.

35.- Woodmansey E J, McMurdo M E T, Macfarlane G T, Macfarlane S. Comparison of compositions and metabolic activities of fecal microbiotas in young adults and in antibiotic-treated and non-antibiotic-treated elderly subjects. Appl Environ Microbiol 2004; 70: 6113-22. doi: 10.1128/AEM.70.10.61136122.2004 .

36.- Drago L, Toscano M, Rodighiero V, De Vecchi E, Mogna G. Cultivable and pyrosequenced fecal microflora in centenarians and young subjects. J Clin Gastroenterol 2012; 46: S81-4. doi: 10.1097/MCG.0b013e3182693982.

37.- Anand R, Song Y, Garg S, Girotra M, Sinha A, Sivaraman A, et al. Effect of aging on the composition of fecal microbiota in donors for FMT and its impact on clinical outcomes. Dig Dis Sci 2017; 62: 1002-8. doi: 10.1007/s10620017-4449-6.

38.- Mariat D, Firmesse O, Levenez F, Guimarăes V, Sokol H, Doré J, et al. The Firmicutes/ Bacteroidetes ratio of the human microbiota changes with age. BMC Microbiol 2009; 9: 123. doi: 10.1186/1471-2180-9-123.

39.- Claesson M J, Cusack S, O'Sullivan O, Greene-Diniz R, de Weerd H, Flannery E, et al. Composition, variability, and temporal stability of the intestinal microbiota of the elderly. Proc Natl Acad Sci U S A 2011; 108: 4586-91. doi: 10.1073/pnas.1000097107.

40.- Kong F, Hua Y, Zeng B, Ning R, Li Y, Zhao J. Gut microbiota signatures of longevity.
Curr Biol 2016; 26: R832-3. doi: 10.1016/j. cub.2016.08.015.

41.- Kelly C R, Kahn S, Kashyap P, Laine L, Rubin D, Atreja A, et al. Update on fecal microbiota transplantation 2015: indications, methodologies, mechanisms, and outlook. Gastroenterology 2015; 149: 223-37. doi: 10.1053/j.gastro.2015.05.008

42.- Mullish B H, Quraishi M N, Segal J P, McCune V L, Baxter M, Marsden G L, et al. The use of faecal microbiota transplant as treatment for recurrent or refractory Clostridium difficile infection and other potential indications: joint British Society of Gastroenterology (BSG) and Healthcare Infection Society (HIS) guidelines. Gut 2018; 67: 1920-41. doi: 10.1136/ gutjnl-2018-316818. 